\title{
A General Approach to Deboronative Radical Chain Reaction with Pinacol Alkylboronic Esters
}

\author{
Emy André-Joyaux, Andrey Kuzovlev, Nicholas D. C. Tappin, Philippe Renaud*
}

University of Bern, department of Chemistry and Biochemistry, Freiestrasse 3, CH-3012

Bern, Switzerland

\begin{abstract}
The generation of carbon-centered radicals from air-sensitive organoboron compounds via nucleohomolytic substitution at boron is one of the most general methods to generate non-functionalized and functionalized radicals. Due to their reduced Lewis acidity, the very popular, air-stable, and readily available alkylboronic pinacol esters are not suitable substrates for this process. Herein, is reported their in situ conversion to alkylboronic catechol esters by boron-transesterification with a substoichiometric amount of catechol methyl borate (MeO-Bcat) telescoped onto a wide array of radical chain processes. This simple one-pot, radical-chain, deboronative protocol allows for the conversion of pinacol boronic esters into iodides, bromides, chlorides, and thioethers. The process is also suitable the formation of nitriles and allylated compounds via $\mathrm{C}-\mathrm{C}$ bond formation using sulfonyl radical traps. Finally, a particularly mild protocol for the protodeboronation of pinacol boronic esters is given. The power of combining radical and classical boron chemistry, is illustrated with a highly modular 5-membered ring formation using a combination of a three-component coupling reaction and a protodeboronative cyclization.
\end{abstract}

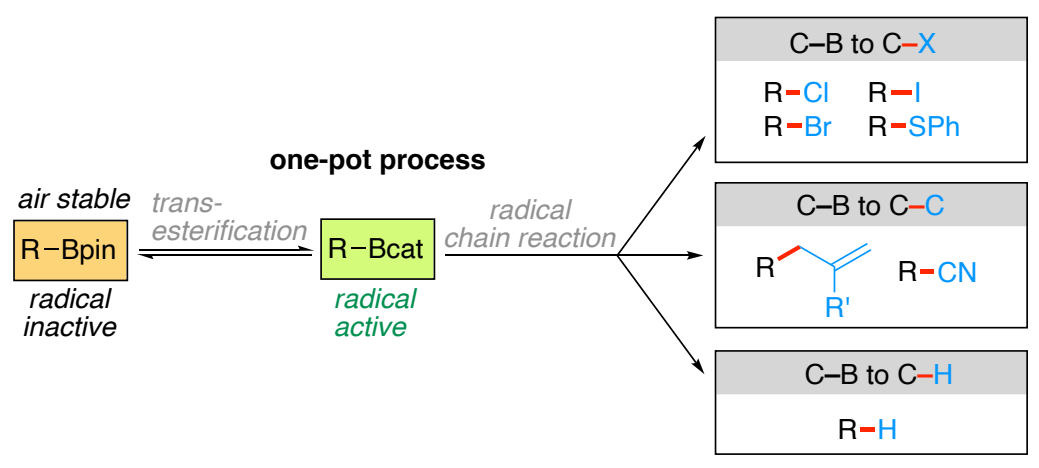

Keywords: Radical reactions, chain reaction, organoboron, deborylation, deboronation, pinacol boronic ester, catechol boronic esters, $B$-alkylpinacolboranes, $B$ methoxycatecholborane, transesterification. 
Radical reactions are becoming routinely applied in research laboratories involved in synthesis ranging from natural products to organic materials. ${ }^{1-3}$ Several approaches have been developed over the years to run radical processes: non-chain reactions based on the persistent radical effect involving homolytic cleavage of a weak bond, ${ }^{4}$ single electron transfer processes using stoichiometric or catalytic redox active agents, ${ }^{5,6}$ photochemistry ${ }^{7,8}$ and chain processes. ${ }^{9-14}$ All these approaches are complementary and have led to significant synthetic methods. Chain reactions are particularly attractive since, beside the reagents, they only require a substoichiometric (often tiny) amount of a radical initiator to take place. However, the mildness of chain processes offers unique opportunities for applications with highly functionalized substrates. For the generation of alkyl radicals in a chain process, alkyl halides, chalcogenides, xanthates and Barton esters have been used as radical precursors.

Organoboron derivatives have also been used successfully for the generation of alkyl radicals ${ }^{15-18}$ via a nucleohomolytic substitution process. ${ }^{19}$ For instance, trialkylboranes provide efficiently alkyl radicals but application of this type of precursor is limited to the generation of simple alkyl radicals since only one of the three alkyl groups at boron is transformed into a radical. ${ }^{20}$ Several years ago, we demonstrated that catechol alkylboronic esters (R-Bcat) are a very efficient source of alkyl radicals. ${ }^{21-23}$ However, more stable and easy to handle boronic acid derivatives such as pinacol alkylboronic ester ( $R-B p i n)$ are radical inactive and therefore not suitable for the direct generation of alkyl radicals in a chain process. The generation of radicals from $\mathrm{R}-\mathrm{Bpin}$ is a highly attractive process that has been tackled in the past by several groups. In an early report, ${ }^{24}$ we have demonstrated that R-Bpin can be directly used in a radical protodeboronation processes by taking advantage of an in situ boron-transesterification with 4-tert-butylcatechol (TBC) catalyzed by sulfuric acid (Scheme 1, A). 


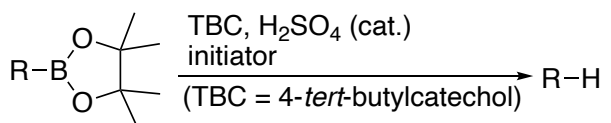

B Lennox and Stahl 2017 - Reaction with TEMPO (electrochemical oxidation)

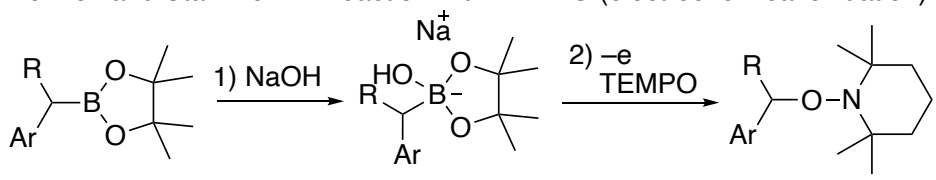

C Aggarwal 2014, Studer 2018 - Protodeboronation (chemical oxidation)

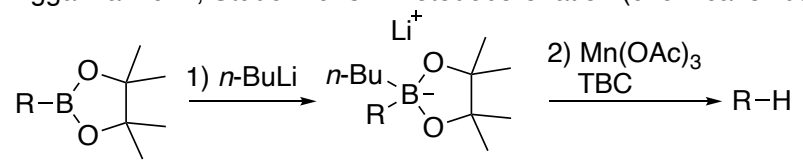

D This work - Reaction with different radical traps (chain reaction)

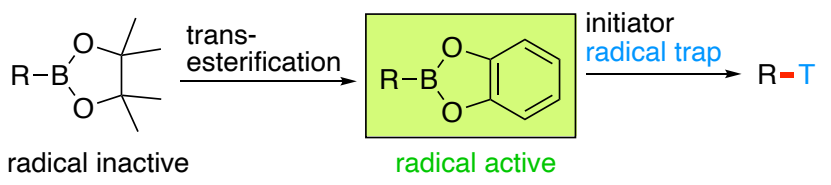

Scheme 1. Strategies for the generation of alkyl radicals from stable pinacol boronic esters.

In this reaction, $\mathrm{TBC}$ has a dual role of i) diol for the transesterification and, ii) hydrogen atom source for the radical chain reaction. This approach suffers from relatively severe reaction conditions, i.e. the use of $20 \mathrm{~mol} \%$ of sulfuric acid at $80{ }^{\circ} \mathrm{C}$, and is limited to protodeboronation. Indeed, TBC used in excess for the transesterification step outcompetes other radical traps by fast transfer of hydrogen atoms to the alkyl radicals. Non-chain approaches such as the generation of radicals via electrochemical oxidation of the ate complexes derived from R-Bpin have been described. ${ }^{25,26}$ For instance, Lennox and Stahl reported the generation of benzylic radicals from $\mathrm{ArCH}(\mathrm{R})-\mathrm{Bpin} \cdot \mathrm{NaOH}$ complexes and were able to trap them with TEMPO (Scheme 1, B). ${ }^{25}$ Similarily, Aggarwal has developed a radical-mediated protodeboronation reaction of $\mathrm{R}-$ Bpin upon treatment with phenyllithium to generate the corresponding ate complexes followed by an oxidative cleavage with $\mathrm{Mn}$ (III) salts in the presence of TBC (Scheme 1, C) ${ }^{27}$ This procedure has also been employed by Studer, ${ }^{28}$ who has recently developed a photoredox catalyzed protodeboronation of ate complexes derived from pinacol boronic ester and $\mathrm{PhLi}$ complexes using thiophenol as a source of hydrogen atoms. ${ }^{29}$ Recently, Ley described an acridinium photocatalyzed oxidative generation of alkyl radicals from pinacol alkylboronic ester - dimethylaminopyridine 
complexes using flow technology. ${ }^{30}$ Other ate complexes such as alkyl trifluoroborates ${ }^{31-61}$ and organo(triol)borates ${ }^{35,36}$ as well as boronic acids ${ }^{30,57,62-65}$ have also been successfully oxidized to deliver the corresponding alkyl radicals using either electrochemical, chemical, or photoredox catalyzed oxidation. ${ }^{66}$ Based on the preliminary results described in Scheme 1A, we report here particularly simple conditions to furnish radicals from a variety of different pinacol alkylboronic esters made possible by an in situ transesterification. This approach is not limited to protodeboronation and a broad range of $\mathrm{C}-\mathrm{X}$ and $\mathrm{C}-\mathrm{C}$ bond forming reactions using a variety of radical traps are possible (Scheme 1, D).

Transesterification. Finding a suitable transesterification method was the key element of our strategy. The most obvious method, i.e. the transesterification with catechol has already been reported by us ${ }^{24}$ but was rapidly abandoned since residual catechol is acting as an excellent source of hydrogen atom in a competitive radical protodeboronation reaction. ${ }^{67,68}$ To circumvent the undesired protodeboronation, alternative procedures for the transesterifications that do not involve free catechol were examined. Transesterification of pinacol alkylboronate $\mathrm{R}$-Bpin with catechol boronic or boric esters was a promising alternative since no free catechol will be present in solution. To avoid the presence of two different radical precursors in the reaction medium, it is important to use radical inactive catechol boronic or boric esters. The boronic esters catechol methylboronic ester (Me-Bcat) and catechol phenylboronic ester (Ph-Bcat) were tested first, both of them are radical inactive, i.e. unable to generate the corresponding highly reactive methyl and phenyl radicals. Under acid catalysis $\left(4 \mathrm{~mol} \% \mathrm{CF}_{3} \mathrm{SO}_{3} \mathrm{H}\right)$, transesterification took place with both boronic esters. However, better results in term of catalyst loading and efficacy were obtained with catechol methyl borate (MeO-Bcat). The transesterification between pinacol 2phenylethylboronic ester 1a and $\mathrm{MeO}-\mathrm{Bcat}$ was investigated in a more systematic way (eq 1). The reaction could be followed by ${ }^{1} \mathrm{H}-\mathrm{NMR}$ and results are summarized in Table 1 (see SI for NMR spectra). In the absence of acid, no reaction took place (entry 1). Trifluoroacetic acid and methanesulfonic acid $(1 \mathrm{~mol} \%)$ were both able to catalyze the transesterification. However, after $4 \mathrm{~h}$, the equilibrium was not reached (Table 1, entries 2-5). Interestingly, trifluoromethanesulfonic acid generated in situ from TMS-triflate and methanol provided a 46:54 mixture of $\mathbf{1} \mathbf{a} / \mathbf{1} \mathbf{a}^{\prime}$ after $2 \mathrm{~h}$ (Table 1, entry 6 ). Longer reaction times did not alter the ratio demonstrating that the thermodynamic equilibrium was reached (Table 1, entry 7). Decreasing the reaction times showed that the equilibrium was reached by 45 minutes (Table 1, entries 8-10). Based on these results, transesterification with $\mathrm{MeO}-\mathrm{Bcat}$ catalyzed by in 
situ generated triflic acid was adopted for the development of the one-pot transesterificationradical process.

Table 1. Transesterification of $1 \mathrm{a}$ with $\mathrm{MeO}-\mathrm{Bcat}$ (1 equivalent).

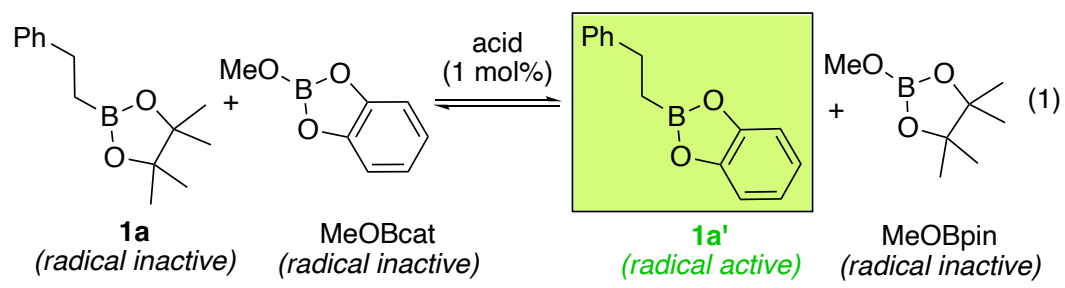

\begin{tabular}{llll}
\hline Entry & Acid $^{\mathrm{a}}$ & Time & $1 \mathrm{a} / 1 \mathrm{a}^{\prime}$ \\
\hline 1 & - & $4 \mathrm{~h}$ & $100: 0$ \\
2 & $\mathrm{CF}_{3} \mathrm{CO}_{2} \mathrm{H}$ & $2 \mathrm{~h}$ & $88: 12$ \\
3 & $\mathrm{CF}_{3} \mathrm{CO}_{2} \mathrm{H}$ & $4 \mathrm{~h}$ & $79: 21$ \\
4 & $\mathrm{CH}_{3} \mathrm{SO}_{3} \mathrm{H}$ & $2 \mathrm{~h}$ & $85: 15$ \\
5 & $\mathrm{CH}_{3} \mathrm{SO}_{3} \mathrm{H}$ & $4 \mathrm{~h}$ & $68: 32$ \\
6 & $\mathrm{CF}_{3} \mathrm{SO}_{3} \mathrm{H}$ & $2 \mathrm{~h}$ & $46: 54$ \\
7 & $\mathrm{CF}_{3} \mathrm{SO}_{3} \mathrm{H}$ & $4 \mathrm{~h}$ & $45: 55$ \\
8 & $\mathrm{CF}_{3} \mathrm{SO}_{3} \mathrm{H}$ & $45 \mathrm{~min}$ & $46: 54$ \\
9 & $\mathrm{CF}_{3} \mathrm{SO}_{3} \mathrm{H}$ & $30 \mathrm{~min}$ & $49: 51$ \\
10 & $\mathrm{CF}_{3} \mathrm{SO}_{3} \mathrm{H}$ & $15 \mathrm{~min}$ & $63: 37$
\end{tabular}

a) Using $1 \mathrm{~mol} \%$ of the acid at $80^{\circ} \mathrm{C}$ in benzene- $\mathrm{d}_{6}$. The ratio of $\mathbf{1 a} / \mathbf{1} \mathbf{a}^{\prime}$ was determined by integrations of the diagnostic $\alpha$-boryl peaks in ${ }^{1} \mathrm{H}$ NMR $(1 \mathrm{a}: 1.26 \mathrm{ppm}(\mathrm{t}, J=8.0 \mathrm{~Hz}, 2 \mathrm{H})$; 1a': $1.41 \mathrm{ppm}(\mathrm{t}, J=8.2 \mathrm{~Hz}, 2 \mathrm{H}))$.

Deboronative halogenation and chalcogenation. The radical bromination of $\mathbf{1 a}$ with benzenesulfonyl bromide initiated by di-tert-butylhyponitrite (DTBHN) ${ }^{69}$ was examined. Gratifyingly, it was immediately observed that only a substoichiometric amount of MeOBcat was necessary to run the reaction to completion (Scheme 2). Using $1 \mathrm{~mol} \%$ of TMSOTf and $\mathrm{MeOH}$ and 0.3 equivalent of $\mathrm{MeOB}$ cat afforded the desired bromide 2a in $68 \%$ yield after 4 h. The yield increased to $95 \%$ after $24 \mathrm{~h}$. Using a smaller amount of $\mathrm{MeO}-\mathrm{Bcat}$ ( 0.1 equiv) was detrimental to the yield. A potential reaction mechanism is provided in Scheme 2 (frame). The radical chain process is coupled with a boron-transesterification reaction. The fact that only a substoichiometric of the radically inactive $\mathrm{MeO}-\mathrm{Bcat}$ is necessary to reach high 
conversion and yields suggest that the $\mathrm{PhSO}_{2}-\mathrm{Bcat}$ generated in the radical chain reaction is also acting as a transesterification reagent.
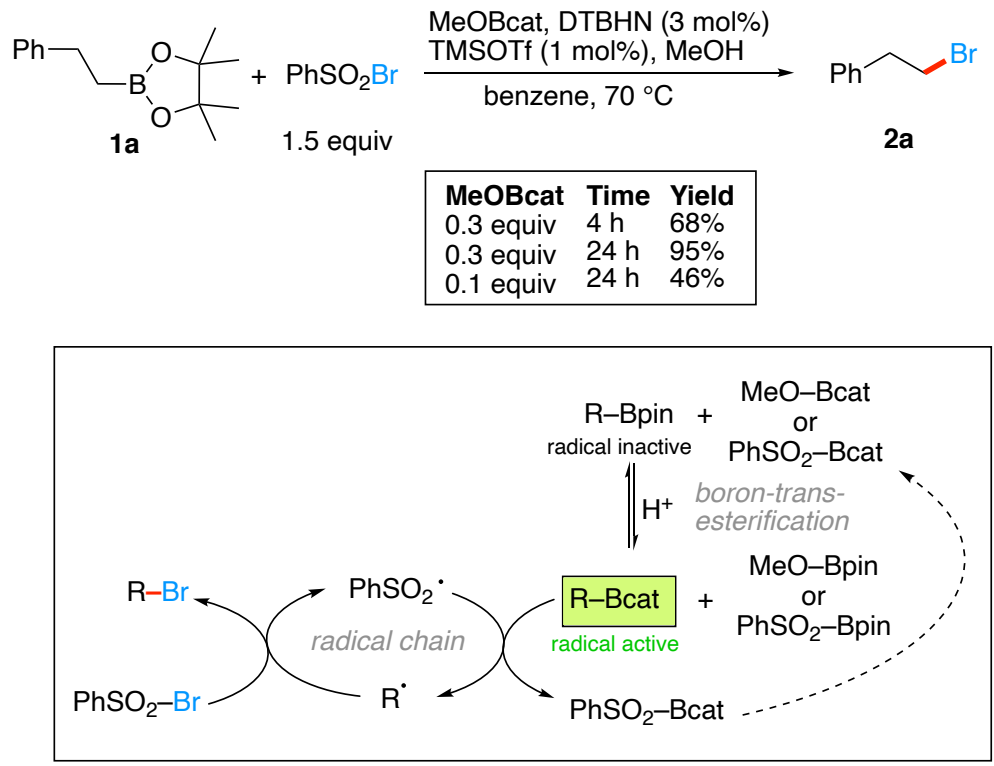

Scheme 2. Optimization of the radical deboronative bromination of $1 \mathrm{a}$.

The optimized reaction conditions were then tested with a variety of substrates and with different halogenating and chalcogenating radical traps such as benzenesulfonyl halides and $S$-phenyl benzenethiosulfonate. The results are summarized in Scheme 3. The reaction worked for a broad range of substrates such as primary $(\mathbf{1 a}-\mathbf{1 c})$ and secondary $(\mathbf{1 d}-\mathbf{1 f})$ alkylboronic esters (Scheme 3, A). Boronic esters delivering stabilized benzylic (1g) and $\alpha$ phosphonyl (1h) radicals provide the expected bromides $\mathbf{2 g}$ and $\mathbf{2 h}$ (Scheme 3, B). The halogenation of radicals derived from $\alpha$-chlorinated pinacol boronic esters $1 \mathbf{i}-\mathbf{1 m}$, easily prepared via Matteson homologation of pinacol alkylboronic ester using lithiated dichloromethane, proceeded efficiently (Scheme 3, C). Overall therefore, this reaction sequence can convert a boryl group into either a dichloromethyl, a bromochloromethyl, or an iodochloromethyl group - three transformations that cannot be achieved easily using known chemistry. The $\alpha$-iodinated boronic ester $\mathbf{1 n}$ was brominated to gem-bromoiodo compound $\mathbf{2 n}$ in $86 \%$ yield. This example demonstrates further that this method is very valuable and general for the preparation of unsymmetrical gem-dihalides (see formation of compounds $\mathbf{2} \mathbf{i}-\mathbf{n}$ ). Finally, the iodination of $\mathbf{1 n}$ provided the symmetrical gem-diiodo derivative $4 \mathbf{n}$ in excellent yield. 

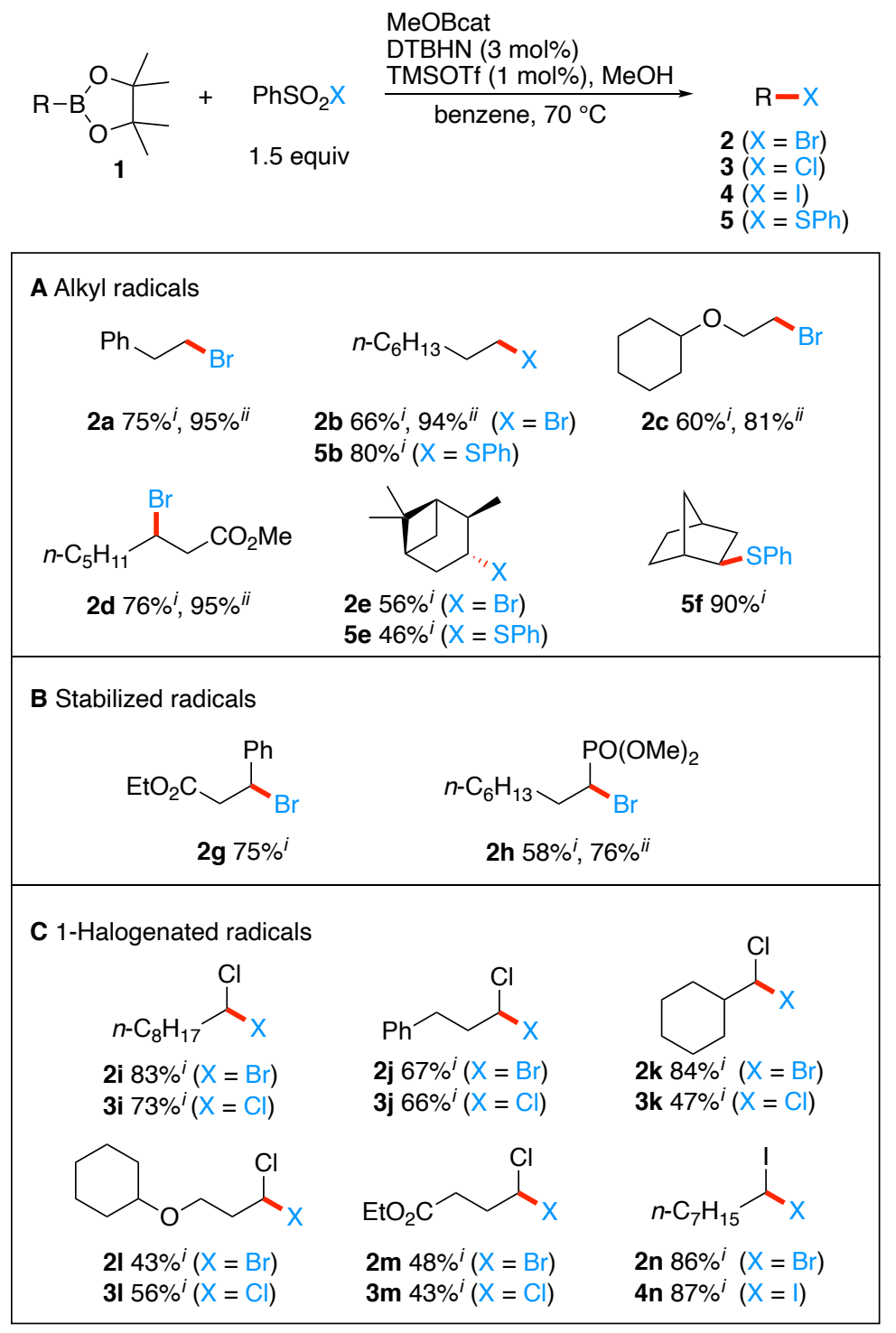

Scheme 3. Deboronative halogenation and chalcogenation of pinacol boronic esters. i) Isolated yield; ii) GC yield.

Deboronative alkylation. Formation of carbon-carbon bonds using different sulfonyl radical traps was investigated next. Simples alkyl radicals derived from 1a-d provided the desired allylated products $\mathbf{6 a}-\mathbf{d}$ in moderate to good yields using 1.5 equivalents of the trap (Scheme $4, \mathbf{A})$. The tertiary $\beta$-keto radical derived from 10 gave $\mathbf{6 0}$ in modest yield. The cyanation of $\mathbf{1 e}$ and 10 proceeded well to give $\mathbf{8 e}$ and $\mathbf{8 0}$, respectively. Stabilized radicals derived from $\mathbf{1 g}$ and $\mathbf{1 h}$ gave the expected allylated products $6 \mathbf{g}, \mathbf{6 h}$, and $7 \mathbf{h}$ in satisfactory yields (Scheme 4, B). Finally, the $\alpha$-chlorinated radicals derived from $\mathbf{1 i}-\mathbf{1 1}$ reacted well with electron deficient and electron rich allylsulfones leading to $\mathbf{6 i}-\mathbf{6 m}$ and $\mathbf{7 j}-\mathbf{m}$, respectively (Scheme $4, \mathbf{C}$ ). The deboronative cyanation of $\mathbf{1 i}$ afforded the $\alpha$-chloronitrile $\mathbf{8 i}$ in $79 \%$ yield. Interestingly, all 
these reactions have been performed using a small excess of the radical trap (1.5 equivalents) leaving room for optimization when structurally more complex organoboranes will be used.
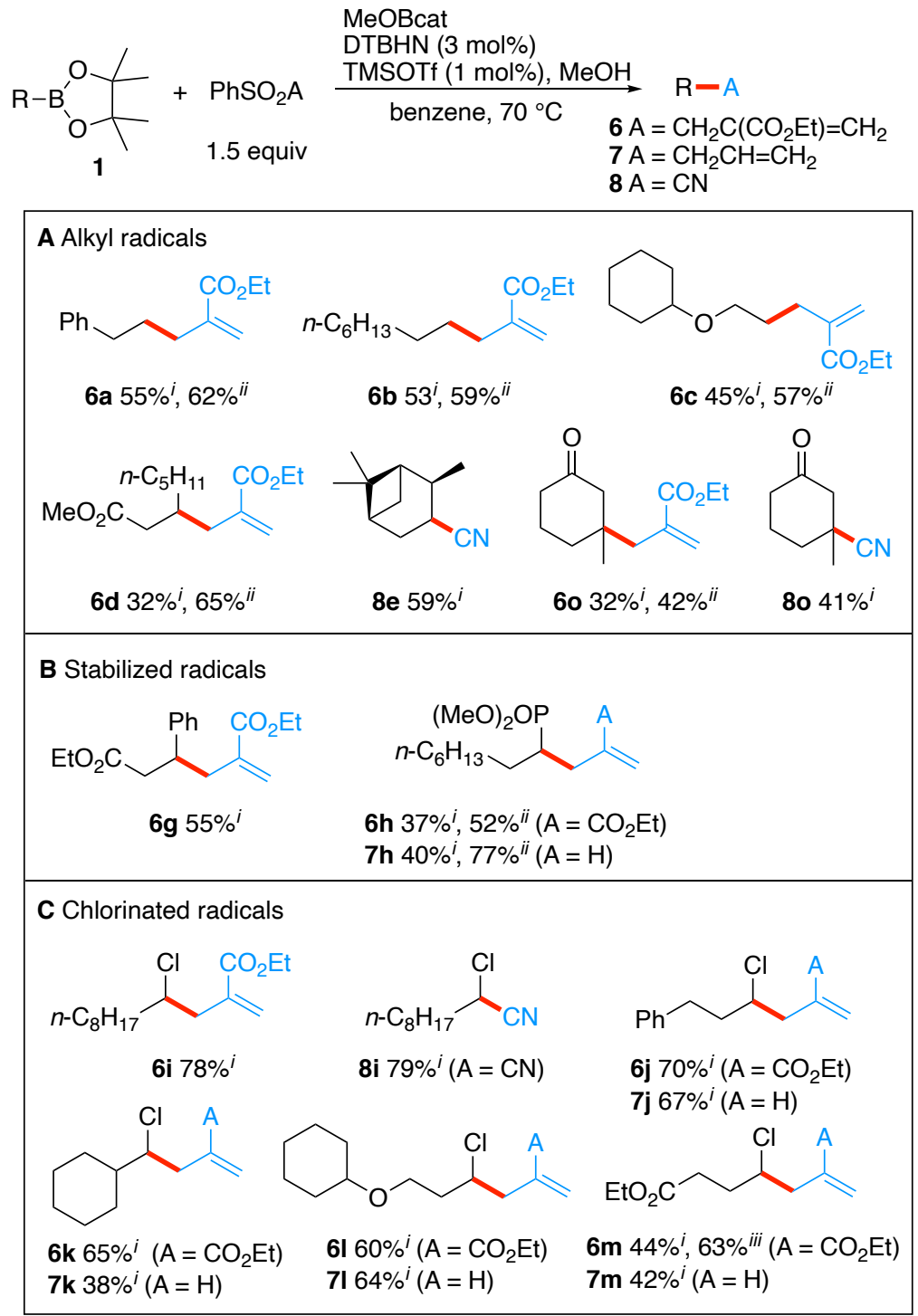

Scheme 4. Deboronative alkylation of pinacol boronic esters using sulfone radical traps. i) Isolated yield; ii) GC yield; iii) ${ }^{1} \mathrm{H}$ NMR yield.

Protodeboronation. Finally, the protodeboronation of pinacol alkylboronic esters was investigated under our mild radical chain reaction conditions using TBC as a source of hydrogen atoms (Scheme 5, conditions a). Under these conditions, primary and secondary alkyl boronates are efficiently converted to the protodeboronated products $9 \mathbf{a}, 9 \mathbf{b}, 9 \mathbf{d}$, and $9 \mathbf{e}$ (Scheme 5, A). The $\alpha$-chlorinated boronates $1 \mathbf{i}-\mathbf{1} \mathbf{m}$ provided the protodeboronated products 9i-9m in high yields. Remarkably, the $\alpha$-iodoboronate $1 \mathbf{n}$ gave $9 \mathbf{n}$ in $89 \%$ yield demonstrating further the very high level of chemoselectivity of this radical 
protodeboronation process. The protodeboronation with TBC on $\alpha$-halogenated boronates $\mathbf{1 i}-$ 1n were run without MeOBcat (condition b). These substrates could be protodeboronated without triflic acid catalysis and only slight reductions in yield. The higher reactivity of the $\alpha-$ haloalkylboronic esters relative to non-halogenated alkylboronic esters is attributed to the higher acidity of the boron atom that facilitates the transesterification.

$$
\begin{aligned}
& \text { a) MeOBcat (0.3 equiv), DTBHN (3 mol\%) } \\
& \text { TMSOTf ( } 1 \text { mol\%), MeOH } \\
& \text { b) DTBHN ( } 3 \text { mol\%), TMSOTf }(1 \mathrm{~mol} \%), \mathrm{MeOH}
\end{aligned}
$$
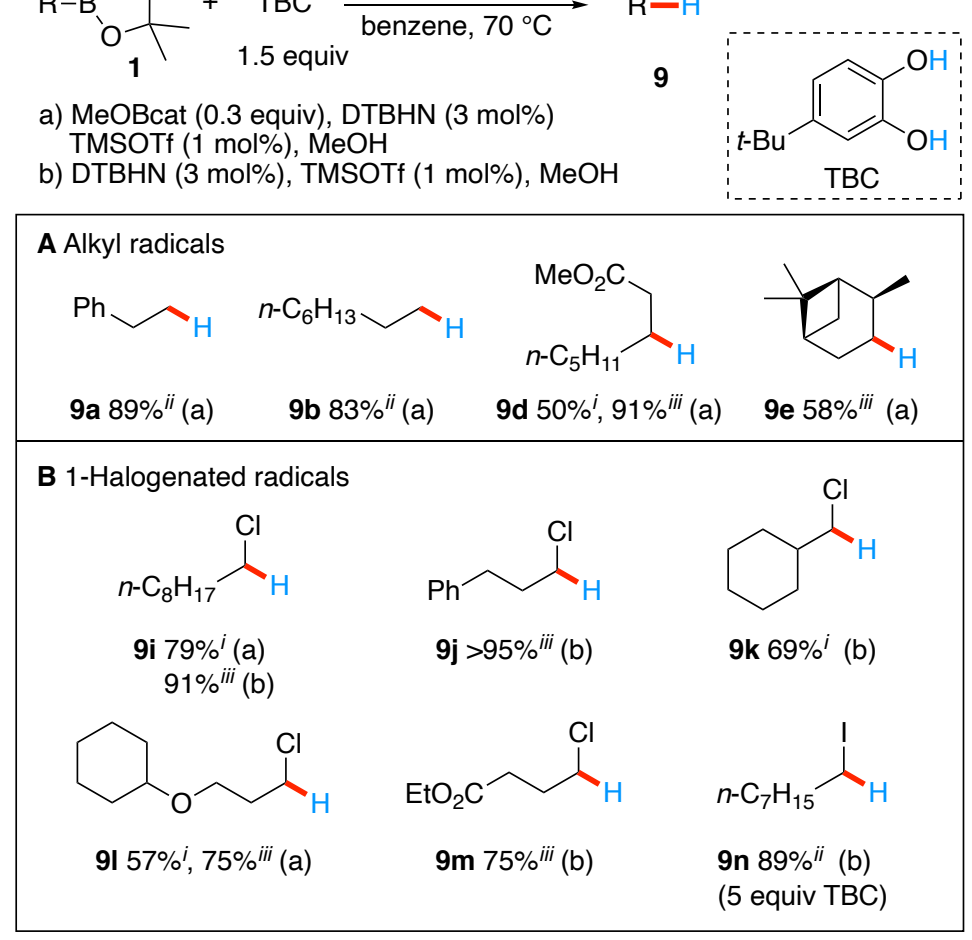

Scheme 5. Protodeboronation of pinacol boronic esters. i) isolated yield; ii) GC yield; iii) ${ }^{1} \mathrm{H}$ NMR yield.

Finally, to demonstrate the potential of merging the rich chemistry of boronic ester with radical chemistry, we have prepared the 5-membered ring 12 via a two-step procedure involving a three-component coupling reaction between dimethyl (3,3-dimethylallyl)malonic acid, pinacol vinylboronic ester and $n$-butyllithium followed by deboronative radical cyclization (Scheme 6). The reported procedure of the three-component coupling process ${ }^{70}$ was significantly improved by avoiding the use of any radical initiator. The chain reaction is presumably initiated by means of an electron transfer from the ate complex to the $\alpha$ bromomalonate. Overall, in the transformation, three new $\mathrm{C}-\mathrm{C}$ bonds and one $\mathrm{C}-\mathrm{H}$ bond are formed. The bonds highlighted in red are formed by radical processes and in blue by an ionic 1,2-metallate (or anionotropic) rearrangement. 


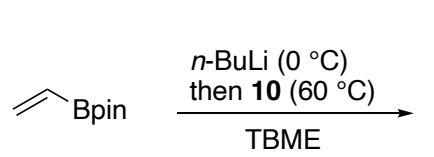

MeOBcat ( 0.3 equiv)

TMSOTf (1 mol\%), MeOH

TBC (1.5 equiv), DTBHN (3 mol\%)

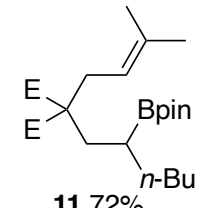

$1172 \%$

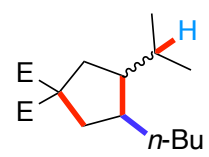

$1246 \%$ (8:2 dr)

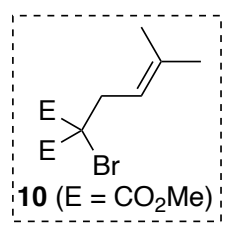

radical

Scheme 6. 5-Membered ring synthesis via merging radical and ionic chemistry of pinacol boronic esters.

Conclusions. We have developed a simple and efficient set of radical chain reactions starting from air stable pinacol alkylboronic esters. Activation of the radical inactive R-Bpin via transesterification with the boric ester $\mathrm{MeO}-\mathrm{Bcat}$ under acid catalysis provides an access to a broad range of radical precursors. The merger of the rich chemistry of boronic esters with radical reactions is expected to open a tremendous number of applications for the synthesis and derivatization of complex target molecules such as natural products and other pharmacologically relevant compounds.

\section{Supporting Information}

Experimental procedures, full characterization of new products, and copies of NMR spectra (PDF)

\section{Corresponding Author}

*Philippe Renaud, University of Bern, Bern, Switzerland;

https://orcid.org/0000-0002-9069-7109; Email: philippe.renaud@dcb.unibe.ch

\section{Author Contributions}

All authors have given approval to the final version of the manuscript. E.A.-J. and A.K. contributed equally.

\section{Notes}

The authors declare no competing financial interest.

\section{Acknowledgment}


The Swiss National Science Foundation (Project 200020_172621) and the University of Bern are gratefully acknowledged for financial support.

\section{References}

1. Renaud, P., Sibi, M. P. \& Wiley InterScience (Online service). Radicals in organic synthesis. (Wiley-VCH, 2001).

2. Encyclopedia of Radicals in Chemistry, Biology and Materials. (John Wiley \& Sons, Ltd, 2012). doi:10.1002/9781119953678.

3. Romero, K. J., Galliher, M. S., Pratt, D. A. \& Stephenson, C. R. J. Radicals in natural product synthesis. Chem. Soc. Rev. 47, 7851-7866 (2018).

4. Leifert, D. \& Studer, A. The Persistent Radical Effect in Organic Synthesis. Angew. Chem. Int. Ed. 59, 74-108 (2020).

5. Zhang, N., Samanta, S. R., Rosen, B. M. \& Percec, V. Single Electron Transfer in Radical Ion and Radical-Mediated Organic, Materials and Polymer Synthesis. Chem. Rev. 114, $5848-5958$ (2014).

6. Prier, C. K., Rankic, D. A. \& MacMillan, D. W. C. Visible Light Photoredox Catalysis with Transition Metal Complexes: Applications in Organic Synthesis. Chem. Rev. 113, 5322$5363(2013)$.

7. Riguet, E. \& Hoffmann, N. Synthetic Radical Photochemistry. Encyclopedia of Radicals in Chemistry, Biology and Materials (2012) doi:10.1002/9781119953678.rad087.

8. Wei, Y., Zhou, Q.-Q., Tan, F., Lu, L.-Q. \& Xiao, W.-J. Visible-Light-Driven Organic Photochemical Reactions in the Absence of External Photocatalysts. Synthesis 51, 30213054 (2019).

9. Giese, B. Syntheses with Radicals-C-C Bond Formation via Organotin and Organomercury Compounds [New Synthetic Methods (52)]. Angew. Chem. Int. Ed. Engl. 24, 553-565 (1985). 
10. Walling, C. Some properties of radical reactions important in synthesis. Tetrahedron 41, 3887 (1985).

11. Barton, D. H. R. \& Zard, S.Z. The invention of high yielding radical chain reactions. NATO ASI Ser. Ser. C Math. Phys. Sci. 189, 443 (1986).

12. Curran, D. P. The design and application of free radical chain reactions in organic synthesis. Part 2. Synthesis 489-513 (1988) doi:10.1055/s-1988-27620.

13. Curran, D. P. The design and application of free radical chain reactions in organic synthesis. Part 1. Synthesis 417 (1988) doi:10.1055/s-1988-27600.

14. Mai, D. N. \& Baxter, R. D. Experimental Strategies for Controlling Radical Chain Reactions. Top. Catal. 60, 580-588 (2017).

15. Ollivier, C. \& Renaud, P. Organoboranes as a source of radicals. Chem. Rev. 101, 34153434 (2001).

16. Darmency, V. \& Renaud, P. Tin-Free Radical Reactions Mediated by Organoboron Compounds. Top. Curr. Chem. 263, 71-106 (2006).

17. Renaud, P. et al. Boron: A key element in radical reactions. Pure Appl. Chem. 79, 223-233 (2007).

18. Renaud, P. Boron in Radical Chemistry. Encyclopedia of Radicals in Chemistry, Biology and Materials vols 2: Synthetic Strategies and Applications 601-628 (2012).

19. Carra, C. \& Scaiano, J. C. Nucleohomolytic Substitution at Boron: A Computational Approach. Eur. J. Org. Chem. 2008, 4454-4459.

20. Brown, H. C. \& Negishi, E. Facile reaction of B-alkylboracyclanes with .alpha.,.beta.unsaturated carbonyl derivatives. Extension of the 1,4-addition reaction via organoboranes to highly branched secondary and tertiary alkyl groups. J. Am. Chem. Soc. 93, 3777-3779 (1971). 
21. Ollivier, C. \& Renaud, P. B-Alkylcatecholboranes as a Source of Radicals for Efficient Conjugate Additions to Unsaturated Ketones and Aldehydes. Chem Eur J 5, 1468-1473 (1999).

22. Ollivier, C. \& Renaud, P. A Convenient and General Tin-Free Procedure for Radical Conjugate Addition. Angew. Chem. Int. Ed Engl. 39, 925-928 (2000).

23. Schaffner, A.-P., Becattini, B., Ollivier, C., Weber, V. \& Renaud, P. BAlkylcatecholboranes as a Source of Radicals for Efficient Conjugate Additions- and Allylations. Synthesis 2003, 2740-2742 (2003).

24. Villa, G., Povie, G. \& Renaud, P. Radical chain reduction of alkylboron compounds with catechols. J. Am. Chem. Soc. 133, 5913-5920 (2011).

25. Lennox, A. J. J., Nutting, J. E. \& Stahl, S. S. Selective electrochemical generation of benzylic radicals enabled by ferrocene-based electron-transfer mediators †Electronic supplementary information (ESI) available: Experimental details, procedures and spectroscopic characterisations. See DOI: 10.1039/c7sc04032f. Chem. Sci. 9, 356-361 (2017).

26. Ohtsuka, K., Inagi, S. \& Fuchigami, T. Electrochemical Properties and Reactions of Oxygen-Containing Organotrifluoroborates and Their Boronic Acid Esters. ChemElectroChem 4, 183-187 (2017).

27. Rasappan, R. \& Aggarwal, V. K. Synthesis of hydroxyphthioceranic acid using a traceless lithiation-borylation-protodeboronation strategy. Nat. Chem. 6, 810-814 (2014).

28. Gerleve, C., Kischkewitz, M. \& Studer, A. Synthesis of $\alpha$-Chiral Ketones and Chiral Alkanes Using Radical Polar Crossover Reactions of Vinyl Boron Ate Complexes. Angew. Chem. Int. Ed. 57, 2441-2444 (2018). 
29. Clausen, F., Kischkewitz, M., Bergander, K. \& Studer, A. Catalytic protodeboronation of pinacol boronic esters: formal anti-Markovnikov hydromethylation of alkenes. Chem. Sci. 10, 6210-6214 (2019).

30. Lima, F. et al. Organic photocatalysis for the radical couplings of boronic acid derivatives in batch and flow. Chem. Commun. 54, 5606-5609 (2018).

31. Nishigaichi, Y., Orimi, T. \& Takuwa, A. Photo-allylation and photo-benzylation of carbonyl compounds using organotrifluoroborate reagents. J. Organomet. Chem. 694, 3837-3839 (2009).

32. Molander, G. A., Colombel, V. \& Braz, V. A. Direct alkylation of heteroaryls using potassium alkyl- and alkoxymethyltrifluoroborates. Org Lett 13, 1852-5 (2011).

33. Sorin, G. et al. Oxidation of Alkyl Trifluoroborates: An Opportunity for Tin-Free Radical Chemistry. Angew. Chem. Int. Ed. 49, 8721-8723 (2010).

34. Molander, G. A., Colombel, V. \& Braz, V. A. Direct Alkylation of Heteroaryls Using Potassium Alkyl- and Alkoxymethyltrifluoroborates. Org. Lett. 13, 1852-1855 (2011).

35. Yasu, Y., Koike, T. \& Akita, M. Visible Light-Induced Selective Generation of Radicals from Organoborates by Photoredox Catalysis. Adv. Synth. Catal. 354, 3414-3420 (2012).

36. Koike, T. \& Akita, M. Visible-Light-Induced Photoredox Catalysis: An Easy Access to Green Radical Chemistry. Synlett 24, 2492-2505 (2013).

37. Miyazawa, K., Yasu, Y., Koike, T. \& Akita, M. Visible-light-induced hydroalkoxymethylation of electron-deficient alkenes by photoredox catalysis. Chem. Commun. 49, 7249 (2013).

38. Huang, H., Zhang, G., Gong, L., Zhang, S. \& Chen, Y. Visible-Light-Induced Chemoselective Deboronative Alkynylation under Biomolecule-Compatible Conditions. $J$. Am. Chem. Soc. 136, 2280-2283 (2014). 
39. Miyazawa, K., Koike, T. \& Akita, M. Hydroaminomethylation of Olefins with Aminomethyltrifluoroborate by Photoredox Catalysis. Adv. Synth. Catal. 356, 2749-2755 (2014).

40. Tellis, J. C., Primer, D. N. \& Molander, G. A. Single-electron transmetalation in organoboron cross-coupling by photoredox/nickel dual catalysis. Science $\mathbf{3 4 5}$, 433-436 (2014).

41. Chinzei, T., Miyazawa, K., Yasu, Y., Koike, T. \& Akita, M. Redox-economical radical generation from organoborates and carboxylic acids by organic photoredox catalysis. $R S C$ $A d v .5,21297-21300(2015)$.

42. Gutierrez, O., Tellis, J. C., Primer, D. N., Molander, G. A. \& Kozlowski, M. C. NickelCatalyzed Cross-Coupling of Photoredox-Generated Radicals: Uncovering a General Manifold for Stereoconvergence in Nickel-Catalyzed Cross-Couplings. J. Am. Chem. Soc. 137, 4896-4899 (2015).

43. Huang, H., Jia, K. \& Chen, Y. Hypervalent Iodine Reagents Enable Chemoselective Deboronative/Decarboxylative Alkenylation by Photoredox Catalysis. Angew. Chem. Int . Ed. 54, 1881-1884 (2015).

44. Karakaya, I., Primer, D. N. \& Molander, G. A. Photoredox Cross-Coupling: Ir/Ni Dual Catalysis for the Synthesis of Benzylic Ethers. Org. Lett. 17, 3294-3297 (2015).

45. Li, Y., Miyazawa, K., Koike, T. \& Akita, M. Alkyl- and aryl-thioalkylation of olefins with organotrifluoroborates by photoredox catalysis. Org. Chem. Front. 2, 319-323 (2015).

46. Primer, D. N., Karakaya, I., Tellis, J. C. \& Molander, G. A. Single-Electron Transmetalation: An Enabling Technology for Secondary Alkylboron Cross-Coupling. $J$. Am. Chem. Soc. 137, 2195-2198 (2015).

47. Verbelen, B. et al. Radical C-H Alkylation of BODIPY Dyes Using Potassium Trifluoroborates or Boronic Acids. Chem. - Eur. J. 21, 12667-12675 (2015). 
48. Yamashita, Y., Tellis, J. C. \& Molander, G. A. Protecting group-free, selective crosscoupling of alkyltrifluoroborates with borylated aryl bromides via photoredox/nickel dual catalysis. Proc. Natl. Acad. Sci. 112, 12026-12029 (2015).

49. Achi, N. E., Penhoat, M., Bakkour, Y., Rolando, C. \& Chausset-Boissarie, L. Continuous UV-Flow Microsystem for Efficient Radical Generation from Organotrifluoroborates by Photoredox Catalysis. Eur. J. Org. Chem. 2016, 4284-4288 (2016).

50. Dai, J.-J. et al. Deboronative cyanation of potassium alkyltrifluoroborates via photoredox catalysis. Chem. Commun. 52, 6793-6796 (2016).

51. El Khatib, M., Serafim, R. A. M. \& Molander, G. A. $\alpha$-Arylation/Heteroarylation of Chiral $\alpha$-Aminomethyltrifluoroborates by Synergistic Iridium Photoredox/Nickel Cross-Coupling Catalysis. Angew. Chem. Int. Ed. 55, 254-258 (2016).

52. Heitz, D. R., Rizwan, K. \& Molander, G. A. Visible-Light-Mediated Alkenylation, Allylation, and Cyanation of Potassium Alkyltrifluoroborates with Organic Photoredox Catalysts. J. Org. Chem. 81, 7308-7313 (2016).

53. Huo, H., Harms, K. \& Meggers, E. Catalytic, Enantioselective Addition of Alkyl Radicals to Alkenes via Visible-Light-Activated Photoredox Catalysis with a Chiral Rhodium Complex. J. Am. Chem. Soc. 138, 6936-6939 (2016).

54. Ryu, D., Primer, D. N., Tellis, J. C. \& Molander, G. A. Single-Electron Transmetalation: Synthesis of 1,1-Diaryl-2,2,2-trifluoroethanes by Photoredox/Nickel Dual Catalytic CrossCoupling. Chem. - Eur. J. 22, 120-123 (2016).

55. Matsui, J. K. \& Molander, G. A. Organocatalyzed, Photoredox Heteroarylation of 2Trifluoroboratochromanones via C-H Functionalization. Org. Lett. 19, 950-953 (2017).

56. Primer, D. N. \& Molander, G. A. Enabling the Cross-Coupling of Tertiary Organoboron Nucleophiles through Radical-Mediated Alkyl Transfer. J.Am.Chem. Soc.139, 9847-9850 (2017). 
57. Xie, S., Li, D., Huang, H., Zhang, F. \& Chen, Y. Intermolecular Radical Addition to Ketoacids Enabled by Boron Activation. J. Am. Chem. Soc. 141, 16237-16242 (2019).

58. Ding, S. et al. Facial strategy for radical species through $\mathrm{Ag}(\mathrm{I})$-mediated oxidation of the alkyl trifluoroborates. Synth. Commun. 48, 936-945 (2018).

59. Ding, S. et al. Silver(I)-mediated Reaction of 2-Isocyanobiaryl with Alkyl Trifluoroborates: Efficient Synthesis of 6-Alkylated Phenanthridines. Chem. Lett. 47, 562-565 (2018).

60. Liu, W., Liu, P., Lv, L. \& Li, C.-J. Metal-Free and Redox-Neutral Conversion of Organotrifluoroborates into Radicals Enabled by Visible Light. Angew. Chem. Int. Ed.57, 13499-13503 (2018).

61. Milligan, J. A., Phelan, J. P., Polites, V. C., Kelly, C. B. \& Molander, G. A. Radical/Polar Annulation Reactions (RPARs) Enable the Modular Construction of Cyclopropanes. Org. Lett. 20, 6840-6844 (2018).

62. Fujiwara, Y. et al. Practical C-H Functionalization of Quinones with Boronic Acids. J.Am. Chem. Soc. 133, 3292-3295 (2011).

63. Iwata, Y., Tanaka, Y., Kubosaki, S., Morita, T. \& Yoshimi, Y. A strategy for generating aryl radicals from arylborates through organic photoredox catalysis: photo-Meerwein type arylation of electron-deficient alkenes. Chem. Commun. 54, 1257-1260 (2018).

64. Li, X., Han, M.-Y., Wang, B., Wang, L. \& Wang, M. Visible-light-induced deboronative alkylarylation of acrylamides with organoboronic acids. Org. Biomol. Chem. 17, 66126619 (2019).

65. Liu, M., Huang, H. \& Chen, Y. Cyclic Iodine Reagents Enable Allylic Alcohols for Alkyl Boronate Addition/Rearrangement by Photoredox Catalysis. Chin.J. Chem. 36, 1209-1212 $(2018)$

66. Duan, K., Yan, X., Liu, Y. \& Li, Z. Recent Progress in the Radical Chemistry of Alkylborates and Alkylboronates. Adv. Synth. Catal. 360, 2781-2795 (2018). 
67. Povie, G. et al. Radical chain repair: The hydroalkylation of polysubstituted unactivated alkenes. Sci. $A d v .4$, eaat6031 (2018).

68. Suravarapu, S. R., Peter, B. \& Renaud, P. Radical-mediated hydroalkylation of 2vinylpyrrolidine derivatives: a versatile entry into indolizidine alkaloids. Sci. China Chem. 62, 1504-1506 (2019).

69. Boukouvalas, J., Cren, S. \& Renaud, P. Di-t-butyl Hyponitrite. in Encyclopedia of Reagents for Organic Synthesis (American Cancer Society, 2007). doi:10.1002/047084289X.rd062.pub2.

70. Tappin, N. D. C., Gnägi-Lux, M. \& Renaud, P. Radical-Triggered Three-Component Coupling Reaction of Alkenylboronates, $\alpha$-Halocarbonyl Compounds, and Organolithium Reagents: The Inverse Ylid Mechanism. Chem. - Eur. J. 24, 11498-11502 (2018). 


\section{Graphical abstract}

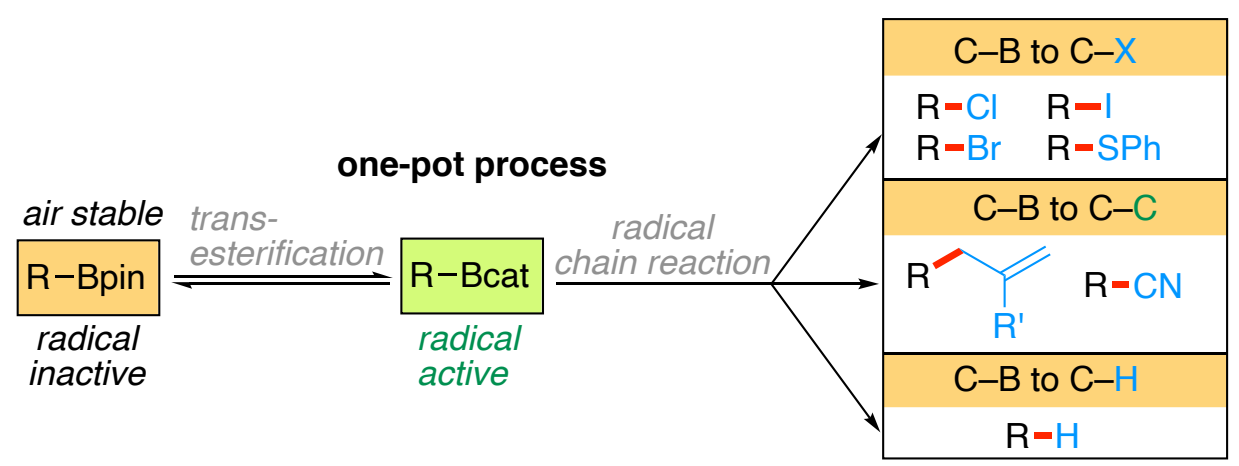

